\title{
Approach to Theoretical Analysis of Processes of Isothermal Straining of Anisotropic High-Strength Materials in Short-Time Creeping Mode
}

\author{
Sergey Sergeevich Yakovlev ${ }^{1}$, Sergey Nikolaevich Larin $^{1} \&$ Valeriy Ivanovich Platonov ${ }^{1}$ \\ ${ }^{1}$ Tula state university, Tula, Russian Federation \\ Correspondence: Sergey Nikolaevich Larin, Tula state university, Tula, Russian Federation. E-mail: \\ mtrushin@mail.ru
}

\author{
Received: October 26, 2014 Accepted: November 6, 2014 Online Published: January 11, 2015 \\ doi:10.5539/mas.v9n3p192 URL: http://dx.doi.org/10.5539/mas.v9n3p192
}

\begin{abstract}
Work Objective. Developing an equation and correlations, which can be used for theoretical analysis of processes of isothermal straining achieved by gas environment and by rigid tools of anisotropic high-strength materials in the mode of short-time creeping. Study Methods The study of isothermal straining processes was performed on the basis of the theory of short-time creeping of anisotropic materials. Study Results. There were developed the principal equations and correlations for theoretical analysis of the kinematics of material flow, stressed and strained state, force conditions, and possibility frontiers for the processes of isothermal straining of anisotropic high-strength materials in the mode of short-time creeping. Conclusions. The equations obtained can be used for theoretical analysis of the processes of isothermal straining achieved by gas environment and by rigid tools of anisotropic high-strength materials in the mode of short-time creeping.
\end{abstract}

Keywords: anisotropy, blank, short-time creeping, damageability, bearing capacity, temperature, stress, strain, destruction, deformation

\section{Introduction}

Improvement of the critical duty item designs determines the application of high-strength hard-to-deform low-ductility materials and manufacture of parts and blocks with special characteristics, which depend on operation conditions. Within the production, complexity of the technological processes requires their long-time processing, which in the final account determines labor intensity and quality of the manufactured items. All these factors call for the need to seek new basic technological processes, for perfection in the methods of analysis and their parameters calculation, as well as thereof duly based approach between the stages of designing and technical preparation for production [1 - 4].

The late practice of manufacturing critical duty parts out of the sheet low-ductility hard-to-strain alloys included the usage of low-speed hot straining featuring not only plasticity, but also creeping nature of the material, and demonstrating large degrees of strain. In the process of slow isothermal straining of these materials depending on the level of excess pressure (gasostatic molding), and on the level of the processing temperature, the values of plastic and creeping strains become commensurate, and this fact should be considered when calculating the technological parameters of the processes [1, 3, $4-9]$.

As a rule, the sheet material exposed to stamping is characterized by certain anisotropy of its mechanical properties, depending on the metal grade and the technological modes of production, which may have a positive or a negative effect upon the stability of the technological processes of metal processing by pressure in various temperature-speed modes $[3,4,10-13,16-20]$.

Mill products used during the metal forming processes possess initial anisotropy of mechanical properties. The mill anisotropy is a consequence of different rolling and heat treatment modes [1-4, 10-15] and, in particular, it depends on the formation of texture of the crystallographic axes' preferred orientation in grains of the processed material, distribution and orientation nature of metal defect phases and retained stresses arising due to the discontinuity of plastic straining under rolling [1-4, 10-15]. During the straining process, grains and inclusions gain an elongated shape, which after annealing transforms into linage structure, whereby the properties, including mechanical ones, can vary dramatically along and across the rolling direction. Studying the kinetics of texture development during cold rolling showed that in general anisotropy grows up with the increase of strain to 
a defined limit, after which it demonstrated almost no changes [1-4, 10-15]. Anisotropy of mechanical properties of a rolled sheet can be reduced by means of texture dispersion relative to the rolling direction.

Anisotropy of mechanical properties of metals demonstrates itself through the differences in yield strengths, ultimate tensile strengths, extension coefficient, and other parameters in different directions of the sheet plane. To characterize the anisotropy, there are used different indicators, for example, anisotropy index of extension coefficient or anisotropy index of ultimate tensile strength. However, these indicators characterize the anisotropy of mechanical properties only along and across the rolling, which is insufficient for an objective assessment of the material anisotropy, as quite a number of materials has the same values of yield strength, ultimate tensile strength, extension coefficient along and across the rolling, but differs in many other ways.

To estimate the anisotropy of mechanical properties of the sheet material, the most commonly used are the anisotropy coefficients, which represent the ratio of logarithmic strains according to the width and thickness of the samples, cut at angles, with respect to the rolling direction at tensile testing. For an isotropic material, this ratio has a unit value. A distinction is made for the transversely isotropic body, when the anisotropy coefficient is practically the same in different directions with respect to the sheet rolling direction, but different from unit value, as well as for the in-plane anisotropy, when the anisotropy coefficient is different in various directions relative to the rolling direction within the sheet plane.

Impact exerted by anisotropy of mechanical properties on formability of sheet materials and the quality of the products obtained are often associated with the value of an average anisotropy coefficient, defined as the arithmetic mean of the anisotropy coefficients in different directions within the sheet plane.

A lot of studies [1-4, 10-15] are devoted to the methodologies handling and experimental determination of the anisotropy coefficients. Typically, the anisotropy coefficient is determined according to the measurement data of the sample's width and thickness obtained in the gauge length zone at the sample's tensile strain by $15 \ldots 20 \%$ within a homogenous strain area. The value is often obtained at a maximum homogenous strain of samples. It is recommended to take a sample's width of at least $15 \mathrm{~mm}$; otherwise there is a tendency to increase the dispersion of value. Instead of thickness measuring, some researchers tried to determine strain value by the length and width data of the sample, and then, using the condition of volume stability, calculated strain by thickness. This method allows eliminating relatively large thickness measurement errors. It is noted that such determination is complicated by an inhomogeneous structure of the material, which leads to distortion of the side surface of the sample, even within the area of semi-uniform strain. It has been established that the coefficient value of a normal anisotropy ranges from 0.2 to 3.5 for the most of sheet materials.

The following works $[1-4,10-15]$ have shown experimentally that the hardening anisotropy takes place under a uniaxial sample tension, i.e. anisotropy coefficient depends on the degree of the sample straining, at which it is determined. The described methods of determining the anisotropy coefficients are characterized by high level of complexity both during the preparation for test, and during the test results processing. In order to remove these shortcomings, there are developed different methodologies for determining the anisotropy of mechanical properties by means of test machines using special mechanical devices in strain blocks.

\section{Methodology}

We have studied straining of an anisotropic material under conditions of short-time creeping. Short-time creeping shall be assumed as a slow straining under conditions of viscous or ductile flow. The elastic components of strain are ignored [1]. We assume that if the value of the equivalent stress [sigma] is less than a certain value $[\text { sigma }]_{\mathrm{e}}$, then the straining process shall occur under conditions of a viscous flow of material, while if [sigma $]_{\mathrm{e}}$ is greater than [sigma $]_{\mathrm{e}}$, then there will be performed a process of straining under conditions of a ductile flow. Value of [sigma $]_{\mathrm{e} 0}$, separating the viscous flow from the ductile flow, corresponds to the material yield point at the degree of strain [epsilon $]_{\mathrm{e} 0}$ and under the test temperature $\mathrm{T}$ determined during static tests of samples.

\section{Main Part}

Let us introduce the potential of strain rates of an anisotropic body at a short-time creeping as follows [1, 3, 4]:

$$
\begin{aligned}
2 f\left(\boldsymbol{\sigma}_{i j}\right)=H & \left(\boldsymbol{\sigma}_{x}-\boldsymbol{\sigma}_{y}\right)^{2}+F\left(\boldsymbol{\sigma}_{y}-\boldsymbol{\sigma}_{z}\right)^{2}+G\left(\boldsymbol{\sigma}_{z}-\boldsymbol{\sigma}_{x}\right)^{2}+ \\
& +2 N \tau_{x y}^{2}+2 L \tau_{y z}^{2}+2 M \tau_{z x}^{2}=1,
\end{aligned}
$$


where $H, F, G, N, L, M$ - are anisotropy parameters for the short-time creeping; [sigma]ij - are the components of the stress tensor; $x, y, z$-are the main anisotropy axes.

In this case the strain rate components [epsilon $]_{\mathrm{ij}}$ are determined according to the associated flow rule $[1,3,4]$. Following the works of R. Hill, and similarly to the theory of orthotropic materials plasticity, let us introduce the notions of equivalent stress $[\text { sigma }]_{\mathrm{e} 0}$ and of the equivalent strain rate [epsilon $]_{\mathrm{e}}$ with deformation under short-time creeping conditions $[1,3,4]$.

Experimental study of the materials' anisotropic properties in various thermo-mechanical conditions have shown that, as a rule, these properties differ under viscous strain (creeping strain) versus ductile strain (creeping-plastic strain). We shall therefore be using index "c" for characteristics of a viscous flow, while the "cp" index will be used for ductile flows.

Thus if a $[\text { sigma }]_{\mathrm{e}}$ value is lower than $[\text { sigma }]_{\mathrm{e} 0}$, then the strain process proceeds under conditions of viscous flow of material, and the value of the equivalent stress [sigma $]_{\mathrm{e}}$ shall be determined as follows $[3,4,13]$ :

$$
\xi_{e}^{c}=B\left(\frac{\sigma_{e}}{\sigma_{e_{0}}}\right)^{n} \quad \text { or } \quad \sigma_{e}=\sigma_{e_{0}}\left(\xi_{e}^{c} / B\right)^{1 / n}
$$

in case when the value of [sigma $]_{\mathrm{e}}$ is larger than $[\text { sigma }]_{\mathrm{e} 0}$, the deformation shall take place under conditions of a ductile flow and the value of [sigma $]_{\mathrm{e}}$ shall be found as follows:

$$
\boldsymbol{\sigma}_{e}=\sigma_{e_{0}}\left(\frac{\varepsilon_{e}^{c p}}{\varepsilon_{e_{0}}}\right)^{m}\left(\frac{\xi_{e}^{c p}}{\xi_{e_{0}}}\right)^{k}, \quad \varepsilon_{e}^{c p}=\int_{t_{0}}^{t} \xi_{e}^{c p} d t,
$$

where $B, n$ - are constants of the material, dependent on the test temperature; [epsilon] e $^{\text {cp }}-$ is the value of the equivalent strain.

It should be noted that, depending on the mark of material and on the processing temperature, we can achieve the strain hardening limit at $\varepsilon_{e}^{c p}=\varepsilon_{e_{1}}^{c p}$ and the strain rate at $\xi_{e}^{c p}=\xi_{e_{1}}^{c p}$, or we can achieve the high-speed hardening limit at $\xi_{e}^{c p}=\xi_{e_{2}}^{c p}$ and strain degree of $\varepsilon_{e}^{c p}=\varepsilon_{e_{2}}^{c p}$.

In order for us to use the above formulas, we should have information about the parameters of the short-time creeping (7) and (8), and about the anisotropy of the mechanical properties of the material under conditions of short-time creeping.

The above parameters can be used for analyzing straining processes occurring under conditions of short-time creeping of orthotropic material.

The limit values of shape changing during metal processing by pressure, performed under various temperature and rate modes of straining, are often estimated based on the phenomenological destruction models. The strain and energy destruction criteria have become the most widely spread ones $[3,4,14,15]$.

Considered were the phenomenological destruction models for accumulating microscopic damages in the process of changing the shape of anisotropic material in the mode of short-time creeping. Let us assume that during the ductile deformation the equivalent strain at the moment of destruction $\mathcal{E}_{e_{n n}}^{c p}$ and the specific fracture work $A_{n p}^{c p}$ depend substantially upon the stress condition index $\sigma / \sigma_{e}$ and upon the relative value of the equivalent rate of straining $\frac{\xi_{e}^{c p}}{\xi_{e_{0}}}$, while in case of viscous straining both $\mathcal{E}_{e_{n p}}^{c}$ and $A_{n p}^{c}$ do not actually depend on those parameters $[3,4,14,15]$; besides, let us take into account the bearing of the first main stress axis versus the main anisotropy axes as determined by angles [alfa], [betta], [gamma]. The effect of the parameter type of the stress condition upon the values $\mathcal{E}_{e_{n p}}^{c p}$ and $A_{n p}^{c p}$ is not taken into account since such information is virtually absent for hot processing. Here $\sigma=\left(\sigma_{1}+\sigma_{2}+\sigma_{3}\right) / 3$ - is the average stress; [sigma]1, [sigma]2 and [sigma] 3 are the main stresses.

It is assumed that in the process of short-time creeping true is the principle of linear superposition of damage summation, taking place in the fields of ductile and viscous deformation. 
We suggest that the conditions for material workability for ductile and viscous flows without destruction are to be formulated respectively as

$$
\omega_{e}^{c p}=\int_{0}^{t} \frac{\xi_{e}^{c p} d t}{\mathcal{E}_{e_{n p}}^{c p}}<1 ; \quad \omega_{e}^{c}=\int_{0}^{t} \frac{\xi_{e}^{c} d t}{\mathcal{E}_{e_{n n}}^{c}}<1,
$$

provided that in these flow ranges true is the deformation fracture criterion, or as

$$
\omega_{A}^{c p}=\int_{0}^{t} \frac{\sigma_{e} \xi_{e}^{c p} d t}{A_{n p}^{c p}}<1 ; \quad \omega_{A}^{c}=\int_{0}^{t} \frac{\sigma_{e} \xi_{e}^{c} d t}{A_{n p}^{c}}<1 .
$$

if the energy fracture criterion is true within these ranges.

Here $[\text { omega }]_{\mathrm{e}}^{\mathrm{cp}}$, $[\text { omega }]_{\mathrm{e}}^{\mathrm{c}}$, and $[\mathrm{omega}]_{\mathrm{A}}^{\mathrm{cp}},[\mathrm{omega}]_{\mathrm{A}}^{\mathrm{c}}-$ is the material damageability at ductile and viscous deformation for the strain destruction model and for the energy destruction model respectively;

$$
\begin{aligned}
\mathcal{E}_{e_{n p}}^{c}=\mathcal{E}_{e_{n p}}^{c}(\alpha, \beta, \gamma) ; & \mathcal{E}_{e_{n p}}^{c p}=\mathcal{E}_{e_{n p}}^{c p}\left(\sigma / \sigma_{e}, \xi_{e}^{n \mathrm{n}} / \xi_{e_{0}}, \alpha, \beta, \gamma\right) ; \\
A_{n p}^{c}=A_{n p}^{c}(\alpha, \beta, \gamma) ; & A_{n p}^{c p}=A_{n p}^{c p}\left(\sigma / \sigma_{e}, \xi_{e}^{n \mathrm{n}} / \xi_{e_{0}}, \alpha, \beta, \gamma\right) .
\end{aligned}
$$

It should be noted that the integration is carried out along the trajectory of the elementary volumes under consideration.

Evaluating the material failure rate for the strain and energy fracture criterions calls for requisite information about the mechanical properties of the material, about the stress and strain states of the elementary volume in the deformation zone, as well as about the magnitudes of the functional dependencies $A_{n p}^{c p}=A_{n p}^{c p}\left(\sigma / \sigma_{e}, \xi_{e}^{n p} / \xi_{e_{0}}, \alpha, \beta, \gamma\right) \quad ; \quad \mathcal{E}_{e_{n p}}^{c p}=\mathcal{E}_{e_{n p}}^{c p}\left(\sigma / \sigma_{e}, \xi_{e}^{n p} / \xi_{e_{0}}, \alpha, \beta, \gamma\right) \quad$ or $\quad A_{n p}^{c}=A_{n p}^{c}(\alpha, \beta, \gamma) \quad$; $\boldsymbol{\varepsilon}_{e_{n p}}^{c}=\boldsymbol{\varepsilon}_{e_{n p}}^{c}(\alpha, \beta, \gamma)$.

Construction of the latter for the studied materials is related to considerable material costs, to the researcher's time, and to availability of the unique testing equipment. The task is considerably simplified if we make use of readily available experimental data for various materials, such as suggested in the available sources $[3,4,14$, $15]$.

Subsequently, when the above mentioned experimental data are available, it is suggested to choose functions $\varepsilon_{e n n n n}^{c p}=f_{1}\left(\sigma / \sigma_{e}, \xi_{e}^{n p} / \xi_{e_{0}}\right)$ and $A_{n n n n}^{c p}=f_{3}\left(\sigma / \sigma_{e}, \xi_{e}^{n p} / \xi_{e_{0}}\right)$ according to the type of the material (aluminum alloys, copper alloys, titanium alloys, steels, high-alloy steels, etc.), while the functional dependencies $\mathcal{E}_{e_{n p}}^{c p}$, $\mathcal{E}_{e_{n p}}^{c}$ or $A_{n p}^{c p}, A_{n p}^{c}$ should be chosen with the help of functions $f_{2}^{c p}(\alpha, \beta, \gamma), f_{4}^{c p}(\alpha, \beta, \gamma)$, $f_{2}^{c}(\alpha, \beta, \gamma), f_{4}^{c}(\alpha, \beta, \gamma)$, which can be determined by way of uniaxial tension of samples cut at angles 0 , 45 and $90^{\circ}$ relative to the rolling direction for cases of plastic and creeping strain.

We consider the anisotropy of the mechanical properties of the blank material for cases of viscous or ductile flow with the help of the following functions: $f_{2}^{c p}(\alpha, \beta, \gamma)=\alpha_{0}+\alpha_{1} \cos \alpha+\alpha_{2} \cos \beta+\alpha_{3} \cos \gamma \quad$ and $f_{2}^{c}(\alpha, \beta, \gamma)=\alpha \mathrm{n}_{0}+\alpha \mathrm{n}_{1} \cos \alpha+\alpha \mathrm{n}_{2} \cos \beta+\alpha \mathrm{n}_{3} \cos \gamma$, where [alfa $]_{0}, \quad$ [alfa] $]_{1}$, [alfa] $]_{2}$ [alfa $]_{3}$ and [alfa]', [alfa]', [alfa]', [alfa]' ${ }_{3}$ - are the experiment constants of the material.

Thus, in order to find the limit value of the equivalent strain for cases of ductile flow, we have the following formulas:

for viscous flow:

$$
\mathcal{E}_{e_{n p} p}^{c p}=C \exp \left(A_{1} \frac{\sigma}{\sigma_{e}}+A_{2} \frac{\xi_{e}^{n p}}{\xi_{e_{0}}}\right) \times\left(\alpha_{0}+\alpha_{1} \cos \alpha+\alpha_{2} \cos \beta+\alpha_{3} \cos \gamma\right)
$$




$$
\varepsilon_{\text {enp }}^{-}=D\left(\alpha \mathbb{C}_{0}+\alpha \mathbb{C}_{1} \cos \alpha+\alpha ?_{2} \quad \beta+\alpha \quad{ }_{3} \quad \gamma\right) .
$$

When studying the destruction criterion in the energy context, we can compute the limit value of the specific fracture work within the field of viscous and ductile strain by using similar formulas through substituting coefficients $C^{\prime}, A_{1}{ }^{\prime}, A_{2}{ }^{\prime}, B^{\prime}, D^{\prime}, \quad b_{i}$ for the coefficients $C, A_{1}, A_{2}, B$, [alfa $]_{\mathrm{i}}$.

Taking account of the damageability when studying the hot straining in the mode of short-time creeping. The issue of the blank destruction in such cases, as it was mentioned above, is considered by taking into account the linear or non-linear accumulation of damages in the strain or in the stress criterion of destruction. However, as it was evidenced by experimental studies $[1,3,4,14,15]$, damaging takes place even in case of minor strains, and it certainly produces its effect upon the stress and the strain conditions.

In view of the above, it is appropriate to introduce damaging into determining correlations (1), (2) and to assume for the chosen law of hardening in the field of ductile flow and in the field of viscous strain, accordingly, that

$$
\boldsymbol{\sigma}_{e}=\boldsymbol{\sigma}_{e_{0}}\left(\frac{\boldsymbol{\varepsilon}_{e}^{n p}}{\varepsilon_{e_{0}}}\right)^{m}\left(\frac{\xi_{e}^{n p}}{\xi_{e_{0}}}\right)^{k}\left(1-\boldsymbol{\omega}^{c p}\right)^{r} ; \boldsymbol{\xi}_{e}^{c}=\frac{B\left(\boldsymbol{\sigma}_{e} / \boldsymbol{\sigma}_{e_{0}}\right)^{n}}{\left(1-\boldsymbol{\omega}^{c}\right)^{d}},
$$

where [omega]cp and [omega]c - are the damages of material for ductile and viscous strains according to the strain ([omega]ecp and [omega]ec) and to the energy ([omega]Acp and [omega]Ac) destruction models respectively; $r$ and $d$-are material constants.

Introduction of the damaging factor into the determining relations within the ductile flow complicates considerably the setting of the task of analyzing the stress and strain states and calls for a simultaneous calculation of the components of stresses, current speeds and strains, equivalent strains or ultimate resiliencies, as well as damaging in the areas of ductile and viscous deformation, but this allows us to obtain a more realistic picture of the deformation.

The criterion of local buckling of sheet blank in the mode of short-term creeping. Many operations of isometric sheet stamping are performed under conditions close to plane stress state, or to plane stress and strained state of the blank. The issue of the limit degrees of strain for plastic straining and straining under creeping flow of isotropic and anisotropic materials, as based on criteria of positive effect of additional loads and of positive effect of the work of additional loads, is discussed in the works [1, 3, 4, 13].

Two-dimensional stress state of the blank. According to the stability postulate offered by Drucker for rheonomic medias, the process of straining under isothermal conditions is infinitesimally stable, if the power of infinitesimally small increments of generalized forces $Q_{i}$ in infinitesimally small increments of the respective velocities of generalized displacements $V_{i}$ is nonnegative $[1,3,4,13]$.

Since negative stability in deformation of the entire body and negative stabilities of the body's parts occur out of step, it is important during the study of technological processes to investigate the localization of deformations in the most stressed elements of the blank.

Let us identify with basic areas a small element of a deformed body. We assume that 1,2,3 - are the principal axes of the Cartesian reference system linked to the element, $a_{i}$ - are the element dimensions along the respective axes, [sigma]i - are the main stresses in the element's facets. Let us use the condition of material incoercibility and consider a rectangular piece of sheet material with thickness $h$, which is subjected to stretching by stresses [sigma]x and [sigma]y, which are the main stresses. Let us assume that coordinate axes $x$, $y, z$ coincide with the main anisotropy axes. The condition of deformation without localizing the deformation is taken down as

$$
\left(\frac{d \sigma_{x}}{d t}-\sigma_{x} \xi_{x}\right)\left(\xi_{x}^{2}+\frac{d \xi_{x}}{d t}\right)+\left(\frac{d \sigma_{y}}{d t}-\sigma_{y} \xi_{y}\right)\left(\xi_{y}{ }^{2}+\frac{d \xi_{y}}{d t}\right) \geq 0
$$

The stress intensity, according to the source [4], is found from 


$$
\begin{gathered}
\sigma_{e}=\sqrt{a_{x}-2 a_{x y} m_{1}+a_{y} m_{1}^{2}} \cdot \sigma_{x}, \\
\text { where } a_{x}=\frac{3 R_{y}\left(R_{x}+1\right)}{2\left(R_{x}+R_{y}+R_{x} R_{y}\right)} ; \quad a_{y}=\frac{3\left(R_{y}+1\right) R_{x}}{2\left(R_{x}+R_{y}+R_{x} R_{y}\right)} ; \quad a_{x y}=\frac{3 R_{x} R_{y}}{2\left(R_{x}+R_{y}+R_{x} R_{y}\right)} .
\end{gathered}
$$

The strain rates $[\mathrm{ksi}]_{\mathrm{x}}$ and $[\mathrm{ksi}]_{\mathrm{y}}$ relate to the strain rate intensity as follows

$$
\xi_{x}=\frac{a_{x}-a_{x y} m_{1}}{\sqrt{a_{x}-2 a_{x y} m_{1}+a_{y} m_{1}^{2}}} \xi_{e}, \quad \xi_{y}=\frac{a_{y} m_{1}-a_{x y}}{\sqrt{a_{x}-2 a_{x y} m_{1}+a_{y} m_{1}^{2}}} \xi_{e},
$$

Using correlations (9) and (10), we simplify (9) to

$$
\left(\frac{1}{z_{1}}-\frac{a \xi_{e}}{A}\right)\left(\frac{a \xi_{e}}{A}+\frac{1}{z_{2}}\right)+m_{1} \frac{b}{a}\left(\frac{1}{z_{3}}-\frac{b \xi_{e}}{A}\right)\left(\frac{b \xi_{e}}{A}+\frac{1}{z_{4}}\right) \geq 0,
$$

where $A=\sqrt{a_{x}-2 a_{x y} m_{1}+a_{y} m_{1}{ }^{2}} ; \quad a=a_{x}-a_{x y} m_{1} ; \quad b=a_{y} a_{x y} m_{1} ; \quad$ and $\quad z_{1}, z_{2}, \quad z_{3}, \quad z_{4}$ - are the values of the subtangents for charts of function dependences $\frac{\sigma_{e}}{A}, \frac{a \xi_{e}}{A}, \frac{m_{1} \sigma_{e}}{A} \frac{b \xi_{e}}{A}$ on time $\frac{1}{z_{1}}=\frac{A}{\sigma_{e}} \frac{d}{d t}\left(\frac{\sigma_{e}}{A}\right)$; $\frac{1}{z_{2}}=\frac{A}{a \xi_{e}} \frac{d}{d t}\left(\frac{a \xi_{e}}{A}\right) ; \quad \frac{1}{z_{3}}=\frac{A}{m_{1} \sigma_{e}} \frac{d}{d t}\left(\frac{m_{1} \sigma_{e}}{A}\right) ; \quad \frac{1}{z_{4}}=\frac{A}{b \xi_{e}} \frac{d}{d t}\left(\frac{b \xi_{e}}{A}\right)$.

Values $z_{1}, z_{2}, z_{3}$ and $z_{4}$ are interconnected by the adopted formula of the equation of state depending on the conditions of straining [6].

\section{Discussion}

The latest practice of manufacturing critical duty parts out of the low-ductility hard-to-strain sheet alloys includes the usage of low-speed hot forming featuring not only plasticity, but also creeping nature of the material, and large strain degrees. During the process of slow isothermal straining of these materials, depending on the level of excess pressure (gasostatic molding) and on the level of the processing temperature, the values of plastic and creeping strains become commensurate, and this fact should be considered when calculating the technological parameters of the processes.

The distinction of this work from the similar ones consists in that the author has developed the basic equations and ratios for the theoretical analysis of the kinematics of the material flow, the stress and strain state, force conditions and possibility frontier of isothermal straining processes taking into account the anisotropy of mechanical properties of the material.

\section{Conclusions}

There have been developed the basic equations and correlations for theoretical analysis of the kinematics of the flow of material, stress and strain condition, power modes and limit possibilities of processes of isothermal deformation of anisotropic high-strength materials in short-term creeping mode. There have been obtained equations of state, phenomenological fracture criteria according to accumulated microdamages and according to local buckling of sheet blanks made out of highly strong anisotropic materials with viscous and ductile flows of material, which can be used for theoretical analysis of the processes of isothermal deformation by gas environment and by strong tool bits of anisotropic highly strong materials in the mode of short-time creeping.

The basic equations and parities are developed for the theoretical analysis of kinematics of a current of the material strained and the deformed condition, power modes and limiting possibilities of processes of isothermal deformation of anisotropic high-strength materials in a mode of short-term creep. Authors receive and result in the given work of the equation of a condition, phenomenological criteria of destruction on accumulation of microdamages and local loss of stability of sheet preparations from high-strength anisotropic materials at viscous and viscous-plastic currents of a material which can be used for the theoretical analysis of processes of isothermal deformation by the gas environment and the rigid tool of anisotropic high-strength materials in a mode of short-term creep. The received results will differ from known decisions with the account of anisotropy 
of mechanical properties of a material which makes essential impact on steady course of considered processes their power and deformation parameters.

\section{Acknowledgement}

His work has been performed within the frameworks of the basic part of the State Task \#2014/227 for performing research and development works of the Ministry of Education and Science of the Russian Federation for the years 2014-2020 and of the RFFI grant \# 14-08-00066 a.

\section{References}

Abbasia, M. et al. (2012). The effect of strain rate and deformation temperature on the characteristics of isothermally hot compressed boron-alloyed steel. Materials Science and Engineering: A. 538, 356-363. http://dx.doi.org/ 10.1016/j.msea.2012.01.060

Alwin, S., Volker, U., Christoph, E., Rainer, K., Alfred, K., Maria, C. M., Roland, R., Wolfgang, S., Domenico, S., \& Dominique, V. (2008). Opportunities and challenges of spray forming high-alloyed steels. Materials Science and Engineering: A., 477(1-2), 69-79. http://dx.doi.org/10.1016/j.msea.2007.08.082

Bogatov, A. A. (2002). Mechanical Properties and Destruction Models for Metals. Yekaterinburg: GOU VPO 'UGTU-UPI', pp: 239.

Chana, K. C., Wanga, G. F., Wanga, C. L., \& Zhang, K. F. (2005). Low temperature superplastic gas pressure forming of electrodeposited Ni/SiCp nanocomposites. Materials Science and Engineering: A. 404(1-2), 108-116. http://dx.doi.org/ 10.1016/j.msea.2005.05.042

Chaoyang, S. et al. (2014). Determination of hot deformation behavior and processing maps of IN 028 alloy using isothermal hot compression test. Materials Science and Engineering, 595, 92-98. http://dx.doi.org/ 10.1016/j.msea.2013.10.051

Chunga, S. W., Higashia, K., \& Kim, W. J. (2004). Superplastic gas pressure forming of fine-grained AZ61 magnesium alloy sheet. Materials Science and Engineering: A. 372(1-2), 15-20. http://dx.doi.org/ 10.1016/j.msea.2003.08.125

Eva-Lis Odenbergera, et al. (2008). Thermo-mechanical material response and hot sheet metal forming of Ti-6242. Materials Science and Engineering: A. 489(1-2), 158-168. http://dx.doi.org/ $10.1007 / \mathrm{s} 12289-012-1094-7$

Fuxiaoa, Yu. et al. (2010). Fundamental differences between spray forming and other semisolid processes. Materials Science and Engineering: A. 304-306, 621-626. http://dx.doi.org/ 10.1016/S0921-5093(00)01547-1

Golenkov, V. A. et al. (2009). Theory of Metal Processing With Pressure. University text-book. M, p. 442.

Grechnikov, F. V. (1998). Straining of Anisotropic Materials. M. Mashinostroyenie, p. 446.

Kolmogorov, V. L. (1986). Mechanics of Metal Processing with Pressure. M. Metallurgia, p. 688.

Li, L., \& Zhang, X. M. (2011). Hot compression deformation behavior and processing parameters of a cast $\mathrm{Mg}-\mathrm{Gd}-\mathrm{Y}-\mathrm{Zr}$ alloy. Materials Science and Engineering: A. 528(3), 1396-1401. http://dx.doi.org/ 10.1016/j.msea.2014.07.025

Malinin, N. N. (1986). Creeping Flow in Metal Processing. M. Mashinostroyenie, p. 216.

Michael, J., O’Briena, F., von Bremenb, H., Furukawac, M., Horitad, Z., \& Terence, G. L. (2007). A finite element analysis of the superplastic forming of an aluminum alloy processed by ECAP. Materials Science and Engineering: A. 456(1-2), 236-242. http://dx.doi.org/ 10.1016/j.msea.2006.11.116

Naderia, M. et al. (2008). Constitutive relationships for 22MnB5 boron steel deformed isothermally at high temperatures. Materials Science and Engineering: A. 478, (1-2), 130-139. http://dx.doi.org/ 10.1016/j.msea.2007.05.094

Puertas, I. et al. (2013). Design and mechanical property analysis of AA1050 turbine blades manufactured by equal channel angular extrusion and isothermal forging. Materials \& Design, 52, 774-784. http://dx.doi.org/ 10.1016/j.matdes.2013.05.089

Romanov, K. I. (1993). Mechanics of Hot Straining of Metals. M. Mashinostroyenie, p. 240.

Rusz, S. et al. (1997). Isothermal plastic forming of high-carbon steel. Materials Science and Engineering: A. 234-236(30), 430-433. 
Testania, C. et al. (2000). AA2618 and AA7075 alloys superplastic transition in isothermal hot-deformation tests. Materials \& Design, 21(4), 305-310.

Yakovlev, S. P. et al. (1997). Processing Anisotropic Materials with Pressure. Kishinev, Quant, p. 332.

Yakovlev, S. P. et al. (2009). Isothermal Pneumatic Molding of Anisotropic High-Strength Sheet Materials. M. Mashinostroyenie, p. 352.

Yakovlev, S. S. et al. (2009). Isothermal Deformation of Anisotropic Materials with Rigid Tools in Short-Time Creeping Mode. M. Mashinostroyenie, p. 412.

Yakovlev, S. S. et al. (2012). Theory and Technology of Stamping Anisotropic Materials. M. Mashinostroyenie, p. 400.

Zhao, W. J. et al., 2013. Isothermal deformation of spray formed Al-Zn-Mg-Cu alloy. Mechanics of Materials, 56, 95-105. http://dx.doi.org/10.4028/www.scientific.net/MSF.788.565

\section{Copyrights}

Copyright for this article is retained by the author(s), with first publication rights granted to the journal.

This is an open-access article distributed under the terms and conditions of the Creative Commons Attribution license (http://creativecommons.org/licenses/by/3.0/). 